Recepción: 24 / 02 / 2017

Aceptación: 18 / 05 / 2017

Publicación: 15 / 07 / 2017

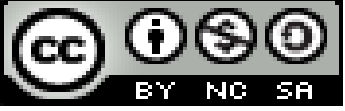

Ciencias económicas y empresariales

Artículo de investigación

\title{
El formador dentro de la Gestión de Recursos Humanos en una empresa
}

\section{The trainer within the Human Resources Management in a company}

\section{O treinador da Gestão de Recursos Humanos em uma empresa}

\author{
Cristian R. Amen-Chinga I \\ cramen@sangregorio.edu.ec \\ Christian A. Vera-Alava II \\ cavera@sangregorio.edu.ec \\ Walter I. Navas-Bayona III \\ winavas@sangregorio.edu.ec
}

Correspondencia: cramen@ sangregorio.edu.ec

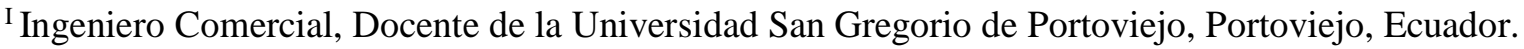

II Magister en Tributación y Finanzas, Diploma Superior en Tributación, Economista, Docente de la Universidad San Gregorio de Portoviejo, Portoviejo, Ecuador.

${ }^{\text {III }}$ Magister en Administración de Empresas, Ingeniero Industrial, Docente de la Universidad San Gregorio de Portoviejo, Portoviejo, Ecuador.
} 


\section{Resumen}

La figura de los formadores en el ámbito empresarial, en este caso de la formación continua o no formal, se está convirtiendo en una necesidad creciente, llámese gestor de formación, tutor, docente, profesor, técnico de formación. Es por ello que este trabajo tiene como objetivo, mostrar algunas consideraciones teóricas en cuanto al formador dentro de la Gestión de Recursos Humanos en una empresa.

Palabras clave: Gestión de recursos humanos; formación continua; capacitación; desarrollo del capital humano; formador.

\section{Abstract}

The figure of trainers in the business field, in this case of continuous or non-formal training, is becoming a growing need, call training manager, tutor, teacher, teacher, training technician. This is why this paper aims to show some theoretical considerations as to the trainer within the Human Resources Management in a company.

Keywords: Human resources management; continuous training; training; development of human capital; trainer.

\section{Resumo}

A figura dos treinadores no campo de negócios, neste caso de treinamento contínuo ou não formal, está se tornando uma necessidade crescente, chamar gerente de treinamento, professor particular, professor, professor, técnico de treinamento. É por isso que este artigo pretende mostrar algumas considerações teóricas quanto ao treinador dentro da Gestão de Recursos Humanos em uma empresa.

Palavras chave: Gestão de recursos humanos; formação contínua; treinamento; desenvolvimento de capital humano; treinador.

\section{Introducción}

Dentro de la Gestión de Recursos Humanos de las empresas, juega un papel protagónico el proceso de formación del personal, y más aún en el este siglo XXI donde la formación continua se direcciona al desarrollo de competencias laborales, y por ende el formador juega un rol fundamental dentro del sistema empresarial. 
El formador debe de poseer competencias específicas para su puesto de trabajo, pero a su vez conocimientos, actitudes y habilidades que le permitan desarrollar competencias en personas que han dejado atrás la etapa escolar. En este caso, los formadores de formación continua necesitan también actividades y procesos de formación encaminados a las competencias pedagógicas y especializadas.

Para garantizar el desarrollo de la formación continua bajo los principios de educación permanente, es preciso considerar algunos aspectos, entre ellos reforzar el papel del formador atendiendo a la mejora de su estatus socio-profesional y de las nuevas competencias que deben asumir (Tejada J., 2010).

Como uno de los actores principales de la formación continua, la figura profesional del formador está llena de exigencias profesionales y personales que probablemente necesitan mayor esfuerzo e imaginación para atender a las demandas diferenciadas de cada persona adulta y grupos de acción en los que éstos se sitúan. Así que el análisis del perfil profesional del formador se presenta un tanto complicado, puesto que en él confluyen una serie de características diversas que surgen y se fomentan en diferentes contextos y/o dimensiones, bajo criterios de la procedencia social, educativa y profesional, así como desde el punto de vista del contexto donde se van a producir las acciones formativas, además de otros factores que tienen que ver con las funciones y roles que tenga que desempeñar en cada situación. (Bonifacio J. J., 1999)

La figura de los formadores en el ámbito de la educación no formal se está convirtiendo en una necesidad creciente, llámese gestor de formación, tutor, docente, profesor, técnico de formación.

La literatura relacionada con la formación continua, ha aportado datos interesantes sobre la tipología del formador de formación continua en las empresas.

Este trabajo tiene como objetivo, mostrar algunas consideraciones teóricas en cuanto al formador dentro de la Gestión de Recursos Humanos en una empresa.

\section{Desarrollo}

El formador es el profesional que en una acción de formación establece una relación pedagógica con los participantes, favorece la adquisición de conocimientos y de competencias, desarrolla 
actitudes y formas de comportamiento, propios a una buena actuación profesional; prepara, desarrolla y evalúa acciones de formación.

El término formador por extensión se refiere a toda persona que de alguna forma o manera tiene relación con la formación desde la óptica de la responsabilidad, de la concepción o de realización de la misma. Comprende un grupo heterogéneo de personas con profesiones muy distintas, difícil de discernir, conceptualizar y de establecer responsabilidades y de asignación de funciones. La distinta procedencia de su formación inicial, su concepción teórica y práctica de la vida y del trabajo, la diversidad de situaciones en que tienen que operar (...), los niveles y modalidades de formación, las especialidades, los materiales que deben emplear o diseñar, la diversidad de grupos destinatarios, no hacen sino añadir complejidad a una profesión nueva y no perfilada en la mayor parte de sus competencias. (Jiménez, 1996).

Consecuentemente, el estudio del perfil profesional de formadores en formación continua no ha logrado hasta hace algunos años acaparar del todo la atención investigadora que merece, porque los estudios realizados hasta ahora no lo contemplan como un objetivo principal sino de apoyo y, en segundo lugar, por la difícil labor de situar este profesional en tareas, competencias y habilidades determinadas, ya que en el contexto de formación continua y del mundo laboral su figura profesional está sujeta a cambios permanentes.

Ferrández (2000), habla de la complejidad de conceptuar, definir su figura y establecer su perfil profesional y argumentan como elementos que lo dificultan, "la falta de deslindamientos en este campo, la ausencia de información en algunas de sus parcelas, la confusión terminológica ante un déficit manifiesto de vocabulario común..." (Ferrández, 2000).

Para Tejada (1999), el formador de formación continua es el profesional que forma adultos ocupados y también futuros formadores y/o docentes, mientras que para Navío (2001) el formador es, sobre todo el profesional de la formación relacionado con el mundo del trabajo. Por lo tanto su referente puede ser tanto profesional como ocupacional. De manera específica desarrolla su actividad en el campo de formación continua.

El formador se relaciona con el especialista que se caracteriza por su experiencia en los contenidos a desarrollar, pero que también domina aspectos pedagógicos. Para ello ha de atender tanto la planificación de la formación que imparte como su desarrollo y evaluación. De forma 
específica, su actividad se circunscribe a la formación continua, al mundo laboral y a los grupos destinatarios (Mamaqi y Miguel, 2011).

Así que la figura del formador de formación continua se conceptualiza bajo los siguientes parámetros:

- El referente concreto de la formación continua, que es una de las especificidades de este profesional y su principal ámbito de actuación.

- Su contexto de actuaciones está delimitado al de las empresas, organizaciones y centros de formación, lo que hace que estas actividades sean aún más específicas.

- Su actuación depende de los grupos destinatarios.

- El perfil del formador de formación continua integra toda una serie de conocimientos, capacidades, destrezas, habilidades y actitudes de definición compleja. (Mamaqi \& Miguel, 2011).

Para Ferrández (2000), el formador también está obligado a "estar en una constante situación de cambio, lo que servía ayer no es válido para hoy porque la tecnología, por ejemplo, encontró una nueva manera de hacer, mediante artilugios más adecuados". Consecuentemente, el formador ha de poseer conocimientos específicos que requieren el desarrollo de diferentes competencias y capacidades; al carácter integral que debe tener la figura del formador se debe añadir la polivalencia, o bien entender de forma global ambos conceptos (capacidades y competencias) bajo el rótulo de "competencias clave". Las capacidades son elementos clave en el estudio de las competencias y ambas son las que permiten hablar de perfiles. Las capacidades no pueden evidenciarse de forma directa en la actividad de los sujetos, ellas se revelan a través de las competencias.

Según Tejada (1999), la competencia significa "el conjunto de saberes (saber, saber hacer, saber estar y saber ser -conocimientos, procedimientos y actitudes) combinados, coordinados e integrados en el ejercicio profesional. El dominio de estos saberes le 'hace capaz' de actuar a un individuo con eficacia en una situación profesional". Así que las capacidades son los recursos que posee una persona representando su potencialidad mientras que las competencias son la movilización de estos recursos definibles en la acción. Los componentes de la competencia son: 
conocimientos, habilidades, capacidades, aspectos prácticos, aspectos éticos, actitudinales, afectivos, volitivos, estéticos y sociales.

En el caso del formador las competencias genéricas se refieren a las competencias teóricas o conceptuales (analizar, comprender, interpretar) integrando el saber y los conocimientos sobre la profesión a desempeñar (conocimientos del contexto general, institucional, aula taller, conocimientos sobre bases psicopedagógicas de la formación, teorías del aprendizaje, conocimiento de los destinatarios, macrodidáctica, microdidáctica, psicopedagogía, orientación, etc.) aplicados desde la planificación de la formación hasta la verificación de los aprendizajes, pasando por las estrategias de enseñanza y aprendizaje, tutoría, monitorización, implicando en ello diferentes medios y recursos didácticos. En las competencias genéricas se incluyen también las competencias sociales (saber relacionarse y colaborar con otras personas de forma comunicativa y constructiva) integrando el saber ser y el saber estar (actitudes, valores y normas). Incluye competencias de organización, administración, gestión, comunicación y animación en la formación (procesos de grupo, trabajo en equipo, negociación, relación interpersonal, liderazgo, análisis estratégico interno y externo, formativo, etc. (Navío, 2005).

Tejada (2010) relaciona las competencias específicas del formador con las que se asimilan bajo las condiciones específicas en las que este profesional desarrolla las competencias. Hoy en día es inconcebible el formador como mero ejecutor de programas de formación. Actualmente se cree en un formador transformador de diseños según su propia situación y su contexto de funcionamiento. Sin embargo, ningún tipo de competencia se puede desarrollar y asimilar fuera de los contextos de actuación del formador sean éstas de carácter genérico y/o específico o de carácter técnico, social, didáctico, etc. Siguiendo este modelo conceptual de contextos se distingue entre:

a) El contexto general: limitado al estatus socio-laboral del formador, considera su grado de profesionalización teniendo en cuenta dos criterios: el laboral (tiempo de dedicación, tipo de contrato, etc.) y el ocupacional (funciones a desempeñar, modalidades de formación que imparten). En cuanto al entorno social, el estudio se ciñe a la titularidad de la institución en la que realiza la formación, la pertenencia a colectivos profesionales, la formación inicial, la titulación máxima, etc. 
b) El contexto específico: limitado al entorno aula-taller, que es el lugar donde se desarrollan las competencias profesionales docentes básicas (planificación, impartición y evaluación de la formación, actitud del formador en el aula y puesto de trabajo, medios didácticos utilizados, rol del formador de aula y en el puesto de trabajo, actividades de orientación y tutoría, etc.) y especificas (conocimientos sobre el mundo laboral, empresarial, del proceso de producción, capacidades, habilidades y actitudes específicas como motivación, poder y autonomía, trabajo en equipo, voluntad de autoperfeccionamiento, etc.).

En el caso del ámbito empresarial se habla de formador, a diferencia del contexto escolar en el que se prefiere la palabra profesor. Con ello nos referimos a aquellos profesionales, con mayor o menor experiencia profesional, que se dedican a organizar y desarrollar la formación a al nivel ocupacional.

"(...) persona que de alguna manera tiene relación con la formación desde la óptica de la responsabilidad, de la concepción o de realización de la misma. Comprende un grupo heterogéneo de personas con profesiones muy distintas, difícil de discernir, conceptualizar y de establecer responsabilidades y de asignación de funciones. La distinta procedencia de su formación inicial, su concepción teórica y práctica de la vida y del trabajo, la diversidad de situaciones en que tienen que operar (...), los niveles y modalidades de formación, las especialidades, los materiales que deben emplear o diseñar, la diversidad de grupos destinatarios, no hacen sino añadir complejidad a una profesión nueva y no perfilada en la mayor parte de sus competencias" (Jiménez, 1996).

"(...) el profesional que forma adultos ocupados y también futuros formadores y/o docentes. (Tejada, 1999).

"(...) es, sobre todo el profesional de la formación relacionado con el mundo del trabajo. Por lo tanto su referente puede ser tanto profesional como ocupacional" (Navío, 2005).

"(...) se relaciona con el especialista que se caracteriza por su experiencia en los contenidos a desarrollar, pero que también domina aspectos pedagógicos. Para ello ha de atender tanto la planificación de la formación que imparte como su desarrollo y evaluación. De forma específica, su actividad se circunscribe al mundo laboral y a los grupos destinatarios (...) Su contexto de 
actuaciones está delimitado al de las empresas, organizaciones y centros de formación, lo que hace que estas actividades sean aún más específicas. (Mamaqi \& Jesús, 2011).

El perfil del formador de formación continua integra toda una serie de competencias de definición compleja que incluyen el analizar, el comprender, el interpretar, el relacionarse, el colaborar, la comunicación, la psicopedagogía, la didáctica, las teorías del aprendizaje. Tienen que tomarse en cuenta las competencias desde la planificación de la formación hasta la verificación de los aprendizajes, pasando por las estrategias de enseñanza y aprendizaje, tutoría, monitorización, implicando en ello diferentes medios y recursos didácticos.

El pedagogo como formador en una empresa, desarrolla tareas relacionados con el diagnostico de necesidades de la empresa, el diseño de planes de formación ya sea a nivel individual o grupal, lleva a cabo la gestión de formación, debe proporcionar los recursos tanto tecnológicos como didácticos necesarios, promueve la innovación y gestiona las futuras prácticas profesionales que se puedan desempeñar en el seno de la empresa. (Moreno García M.S., 2009).

Ahora bien, no se puede concebir el formador sólo como ejecutor de programas de formación, sino ser un especialista según su propia situación y su contexto de funcionamiento.

Asenjo (2005), plantea que en este contexto cambiante el formador se enfrenta ante importantes retos y debe adaptarse a las nuevas realidades entre ellas menciona: las nuevas tecnologías (cañón de luz, pizarra digital, e-mail, webs de la asignatura, foros, entre otros) y su participación como diseñador y tutor en formaciones online (web based training) y off line (computer based training); el desarrollo de la inteligencia emocional a través de formaciones vivenciales y análisis de las propias emociones y las de los grupos para obtener mejores resultados profesionales y empresariales; el desarrollo y extensión de la formación a medida más próxima a las necesidades formativas, para facilitar el desarrollo de competencias profesionales y su transferencia a los puestos de trabajo y las cuentas de explotación de las empresas; el formador ha de estar al día en los conceptos teóricos, pero simultáneamente dominar su aplicación práctica. Además, los roles del formador se han extendido, ya que sobrepasan el de facilitador de aprendizajes, alcanzando otros estadios más elevados como el de consultor, consejero, coach y terapeuta entre otros.

Si nos centramos en la formación en la empresa, no se puede olvidar que este tipo de centros o instituciones laborales se articula, se organiza y se gestiona sobre diferentes parámetros de 
referencia, fundamentalmente el de la producción, por lo que la formación se convierte en una estrategia o necesidad a tal fin.

En este caso, podemos encontrar al menos dos tipologías según el tamaño de la empresa o institución socio laboral (grande o pequeña); es decir, si pueden disponer de un departamento específico de formación en su seno o no. Esta última situación llevaría a demandar la formación de formadores a otras instituciones (universidad, consultorías, entidades colaboradoras, etc.).

Cuando se trata de grandes empresas que cuentan con departamento de formación, además de la oferta formativa específica de cursos de formación profesional y ocupacional, ofertan cursos de preparación de formadores de carácter específico, aunque pueden plantearse situaciones de formación en cascada y contratar servicios de formación externa para cursos de carácter más general. Dado el carácter de la formación muy aplicada al puesto de trabajo, la relación con otros profesionales de la institución es muy estrecha, haciéndoles partícipes a su vez de las modalidades de formación en el puesto de trabajo (coaching, mentoring, instructor, etc) (...) Por el contrario, cuando se trata de pequeñas empresas, se suele recurrir a departamentos universitarios o consultoras externas que vienen a llenar el vacío del departamento de formación. (Giménez Marín V., 2011).

Son varias las propuestas que concretan lo que en la actualidad le incumbe al formador en forma de competencias. Los planteamientos son diversos y van desde lo más general a lo más específico. La coincidencia estriba en que el perfil del formador es mixto, es decir que necesita tanto competencias pedagógicas como competencias profesionales afín a su temática de intervención.

Navío (2001) cita a Tejada (2001) al proponer las siguientes competencias para el formador:

1. Competencias teóricas o conceptuales que integren el saber de la profesión y el saber hacer cognitivo.

2. Competencias psicopedagógicas y metodológicas que integren el saber y el saber hacer. Esto alcanza desde la planificación hasta la verificación de los aprendizajes, pasando por las estrategias de enseñanza-aprendizaje, tutorías, etc. 
3. Competencias sociales que integran el saber ser y el saber estar. Esto supone aspectos de organización, administración, gestión, comunicación y animación de la formación.

Y Navío (2001) considera que:

1. No existe un profesional de la formación continua cuyas competencias básicas sean diferentes respectos a otros profesionales de la formación, por lo tanto el formador de formación continua asume una serie de competencias específicas definidas por el contexto en el que actúa.

2. Los distintos profesionales relacionados con la formación que intervienen en el contexto de la formación continua asumen, en función del contexto en el que actúan y en función de los destinatarios, diferentes especializaciones donde se manifiestan diferentes combinaciones en la asunción de funciones.

3. No todos los profesionales que actúan como formadores en el contexto de la formación continua pueden ser considerados como profesionales de la formación. Al respecto, cabe diferenciar aquellos que dedican su actividad principal a la formación respecto a los que siendo profesionales de otro ámbito distinto al de la formación, realizan tareas de formación en momentos puntuales. (Navío Gámez A., 2001).

\section{Conclusiones}

En las empresas, el papel del formador dentro de la Gestión de Recursos Humanos, está cada vez siendo más importante, y más cuando las normas ISO 9001 incluyen el proceso de formación del personal.

A partir de las exigencias del sistema de calidad, por un lado este formador se involucra en el proceso de gestión de necesidades de formación, que incluye el identificar necesidades de formación (diagnóstico de necesidades de la empresa), planificar la formación (diseño de planes de formación) y evaluar la formación. Así como proporcionar los recursos, tanto tecnológicos como didácticos, necesarios y promoviendo la innovación en la planificación y desarrollo de tareas formativas. Y por otro lado el especialista que asume la ejecución de los entrenamientos en el puesto de trabajo, la tutoría de los nuevos empleados, los seminarios para un mejor desempeño. 


\section{Referencias bibliográficas}

Asenjo, J. (2005). El perfil del formador ante los retos de la formación, IV Congreso de formación para el trabajo. Zaragoza.

Bonifacio J. J. (1999). Los formadores. Educar, n. 20.

Ferrández, A. A. (2000). La formación ocupacional en el marco de formación continua de adultos. Formación y Empleo: enseñanza y competencias. Granada: Comares.

Giménez Marín V., T. J. (2011). Formación de formadores. Tomo 1. Escenario aula.

Jiménez, B. (1996). Claves para comprender la formación profesional en Europa y en España Barcelona.

Mamaqi, X., \& Jesús, M. (2011). El perfil profesional de los formadores de formación continua en España. RELIEVE, 17.

Moreno García M.S. (2009). La empresa: un reto para los profesionales de la pedagogía. Cuestiones Pedagógicas, 20.

Navío, A. (2005). Características de una formación de formadores basada en competencias. Actas do VI Congresso Internacional de Formaçao para o Trabalho Norte de Portugal/Galiza. Portugal. Navío Gámez A. (2001). Las competencias del formador de formación continua. Análisis desde los programas de formación de formadores. Universidad autónoma de Barcelona, España.

Tejada, J. (1999). Acerca de las Competencias Profesionales [ElectronicVersion]. Revista Herramientas núm. 57, fromhttp://dewey.uab.es/pmarques/dioe/competencias.pdf Tejada J. (2010). Profesionalización docente en el escenario de la Europa de 2010. Una mirada desde la formación. Revista de Educación, v. 349. 